\title{
Electronic Health Records and Environmental Public Health Tracking
}

\author{
Gonza Namulanda* \\ Centers for Disease Control and Prevention, Atlanta, GA, USA
}

\section{Objective}

This presentation will discuss how the Tracking Network is exploring the use of EHR to meet Tracking Network surveillance challenges and provide other opportunities to enhance environmental public health surveillance

\section{Introduction}

The Environmental Public Health Tracking Network (Tracking Network) is a national surveillance system that integrates environmental hazard, exposure, and health outcome data into one system. The Tracking Network launched in July 2009, and has since been receiving data from 23 funded state and local health departments, and several national partners, e.g., Environmental Protection Agency. Despite this success, some challenges exist in obtaining more timely and complete data to link risk factors, and assign exposure for health outcomes with long latency periods before their detection.

The Health Information Technology for Economic and Clinical Health (HITECH) Act (2009) facilitates the adoption of electronic health records (EHR) through incentivizing the meaningful use of certified EHR Technology. Meaningful use is a set of specific objectives and data exchange standards that eligible healthcare professionals and hospitals must achieve to qualify for the Centers for Medicare and Medicaid Services (CMS) Electronic Health Records Incentive Programs. Public health agencies in turn need to have the capacity to accept these data in the mandated standard and determine the potential and use of this increased data.

This presentation will discuss how the Tracking Network is exploring the use of EHR to meet Tracking Network surveillance challenges and provide other opportunities to enhance environmental public health surveillance. We will also present some results of these efforts.

\section{Methods}

The Tracking Network initiated an exploratory project with the goals of determining: i) the data that the Tracking Network can use from EHR; ii) information that can be included in EHR to enhance environmental public health surveillance; and iii) potential use of Tracking Network data in an EHR e.g., for community health needs assessments.

We interviewed eight public health informaticians, public health professionals, and disease surveillance experts. We also participated in CDC's EHR initiatives such as CDC and Office of the National Coordinator for Health Information Technology's (ONC) Standards and Interoperability (S\&I) Framework initiatives namely, Structured Data Capture (SDC) whose aim is to define requirements and standards to facilitate the collection of supplemental EHR-derived data, and Data Access Framework (DAF) whose aim is to facilitate the query of aggregate data from EHR.

\section{Results}

Through this work, we have been able to prioritize data that the Tracking Network could utilize from EHR; identify a list of possible partners for a pilot project to further develop and test how these data can be reported from an EHR; identify priority supplemental data that can be added to EHR to enhance environmental public health surveillance; and develop use-cases for the SDC and DAF initiatives.

\section{Conclusions}

Electronic health records have the potential to enhance public health surveillance by providing more comprehensive, accurate and timely data. However several challenges exist such as: policy and data privacy issues, and the lack of common data elements among others.

\section{Keywords}

Environmental public health tracking; Environmental public health surveillance; Electronic health records; Meaningful use

\section{Acknowledgments}

We would like to thank the Tracking Program grantees and contractors who contributed to this work, all the experts who allowed us to interview them, and participants on the S\&I framework initiatives.

\section{*Gonza Namulanda}

E-mail: fos0@cdc.gov 